\title{
Management of Patients under Treatment with Monoclonal Antibodies and New Biological Therapies
}

\author{
Marta Amigo-Basilio (D, Covadonga Álvarez-González, Carlos Cobo-Vázquez *D, Isabel Leco-Berrocal, \\ Luis Miguel Sáez-Alcaide and Cristina Méniz-García (D)
}

Department of Medicine and Orofacial Surgery, Faculty of Dentistry, Complutense University of Madrid, 28040 Madrid, Spain; mamigo@ucm.es (M.A.-B.); covaalva@ucm.es (C.Á.-G.); mileco@odon.ucm.es (I.L.-B.); 1saez@ucm.es (L.M.S.-A.); cmenizga@ucm.es (C.M.-G.)

* Correspondence: drcarloscobo@gmail.com; Tel.: +61-2-9351-3391

check for updates

Citation: Amigo-Basilio, M.;

Álvarez-González, C.; Cobo-Vázquez,

C.; Leco-Berrocal, I.; Sáez-Alcaide,

L.M.; Méniz-García, C. Management of Patients under Treatment with Monoclonal Antibodies and New Biological Therapies. Appl. Sci. 2021, 11, 4865. https://doi.org/10.3390/ app11114865

Academic Editor: Francesco Cappello

Received: 28 April 2021

Accepted: 22 May 2021

Published: 25 May 2021

Publisher's Note: MDPI stays neutral with regard to jurisdictional claims in published maps and institutional affiliations.

Copyright: (c) 2021 by the authors. Licensee MDPI, Basel, Switzerland. This article is an open access article distributed under the terms and conditions of the Creative Commons Attribution (CC BY) license (https:/ / creativecommons.org/licenses/by/ $4.0 /)$.

\begin{abstract}
Objective: The aim of this study is to know the biological therapy drugs that are related to adverse events, what dental treatments are associated with the appearance of these events, their severity, and how they are resolved. Study design: Analysis of cases described in the literature on patients undergoing treatment with biological therapies who have developed adverse effects associated with these drugs. Results: Of the 62 articles reviewed, 49 describe 68 cases of MRONJ, most of which appeared in the jaw and received surgical and/or conservative treatment. Conclusions: Biological therapies can potentially develop adverse effects in the oral cavity, so strict monitoring by the dentist is necessary.
\end{abstract}

Keywords: target therapy; antiangiogenic agent; denosumab; osteonecrosis of the jaw; monoclonal antibody; stomatitis; dental infection; MRONJ

\section{Introduction}

Biological therapies are a new method of treating some autoimmune diseases and cancer. They were defined by the FDA (Food and Drugs Administration) as any virus, therapeutic serum, toxin, antitoxin, or similar product applicable to the prevention and treatment of human diseases. These biologics include vaccines, blood and blood-derived preparations, antitoxins, growth hormones, human insulin, gene therapies, recombinant therapies, proteins, and allergens, along with the newer biologics: cytokines, monoclonal antibodies, and fusion proteins [1,2].

Cytokines are immunomodulators that regulate the host's response to inflammation and infections. In this way they work by signaling molecules that will bind to protein receptors on cells, generating a physiological change in them.

Monoclonal antibodies are synthetic molecules that activate against certain antigens to improve immune recognition. They are divided into four subgroups: murine, chimeric, humanized, and human.

Fusion proteins are composed of transmembrane proteins connected to another molecule through the Fc portion of human immunoglobulin.

The mechanisms of action of biological therapies involve a series of physiological changes that can trigger side effects in the patient. One of these mechanisms is the inhibition of molecules such as $\mathrm{TNF} \alpha$, which are essential in the inflammatory response. This produces a change in the immune system that can lead to these patients having a greater predisposition to infections [1].

Other mechanisms of action to take into account, due to their physiological repercussions, are antiresorptive and antiangiogenic, influencing osteoclasts and vascular endothelial growth factor (VEGF), respectively $[2,3]$.

Despite the efficacy and safety of these drugs in the vast majority of patients, there is a significant risk of producing adverse effects such as mucositis, hyperkeratotic lesions, 
mucosal dyschromia, geographic tongue, dysgeusia, lichenoid lesions, telangiectasias, xerostomia, dysesthesia, and, the most prominent, osteonecrosis of the jaw, due to its antiangiogenic and/or antiresorptive power [4]. This osteonecrosis will be considered related to these medications when the following characteristics described by the AAOMS (American Association of Oral and Maxillofacial Surgeons) are present in a patient:

- Previous or current treatment with antiresorptive or antiangiogenic agents.

- Exposed bone or bone that can be probed through an intra- or extra-oral fistula in the maxillofacial region, which has persisted for more than 8 weeks.

- Absence of radiotherapy or previous metastasis in the jaw [2].

In 2014, the AAOMS changed the term "Bisphosphonate-Related Osteonecrosis of the Jaws" (BRONJ) to "Medication-related Osteonecrosis of the Jaws" (MRONJ) to include all other drugs related to osteonecrosis. However, since 2017 there are only three monoclonal antibodies officially recognized by the FDA as causing MRONJ: denosumab, bevacizumab, and sunitinib. The rest of the reported drugs have not yet been recognized as such due to the lack of scientific evidence [5].

The objective of this study is to know the drugs related to adverse events in oral surgery, what effects they produce, and to record how they have been resolved according to the available literature.

\section{Material and Methods}

An electronic search was carried out in the National Library of American Medicine (MEDLINE) through Pubmed, with the combination of the following terms: "MRONJ"; "Adverse effect" AND "dental disease" AND "targeted therapy", applying the results filters by year between 2005 and 2020, in humans, and with the full text available.

The following inclusion criteria were established: articles published since 2005, but due to the lack of described literature on the subject, the search was extended to those published since 2005; in English or Spanish; performed in humans; patients being treated with biological therapies; and in relation to the objective of this review. Following the exclusion criteria, those articles published prior to 2005 were eliminated from the search; in a language other than Spanish or English; performed on animals; those duplicates; and those that, after reading the abstract, were not related to the main objective of this review.

\section{Results}

A total of 374 articles were obtained, to which the criteria described above were applied, going to read full text 83 articles, and finally selecting a total of 54 for this review (Figure 1).

Within our selection, a total of 49 articles have been compiled in relation to osteonecrosis of the jaws [5-22], two articles in relation to lesions in the oral mucosa [4,23], two with oral infections [24,25], and, finally, one article reporting a case of exudative erythema multiforme [26] (EEM) associated with the use of biological therapies.

Of the total of 49 articles related to osteonecrosis of the jaws, 14 cases were collected that refer to bevacizumab [10,27-39], eight cases to sunitinib [17-22,29,39], six cases to denosumab [10-14] and everolimus [29,39-43], five cases to rituximab [5,11-14], four cases to temsirolimus $[14,26,29,34]$, three cases referred to sorafenib $[19,29,33]$ and aflibercept [44-46], two cases related to trastuzumab and pertuzumab [15,39], imatinib [39,47], and pazopanib [39,42]. Finally, only one article was found referring to tacrolimus [5], lenvatinib [16], erlotinib [33], ipilimumab [48], cabozantinib [49], afatinib [39], nilotinib [39], dasatinib [39], or tocilizumab [50].

Regarding the treatment carried out for osteonecrosis, in a total of 38 cases a surgical treatment was carried out, in 23 cases a conservative treatment was carried out, and in seven of them the treatment followed was not specified.

The surgical act is the triggering factor reported in most cases, with a total of 27; this is followed by a poor fit of the prostheses, mentioned in five cases; finally, in 31 the triggering 
factor for osteonecrosis is not described and in the remaining five cases a factor other than those previously mentioned is related.

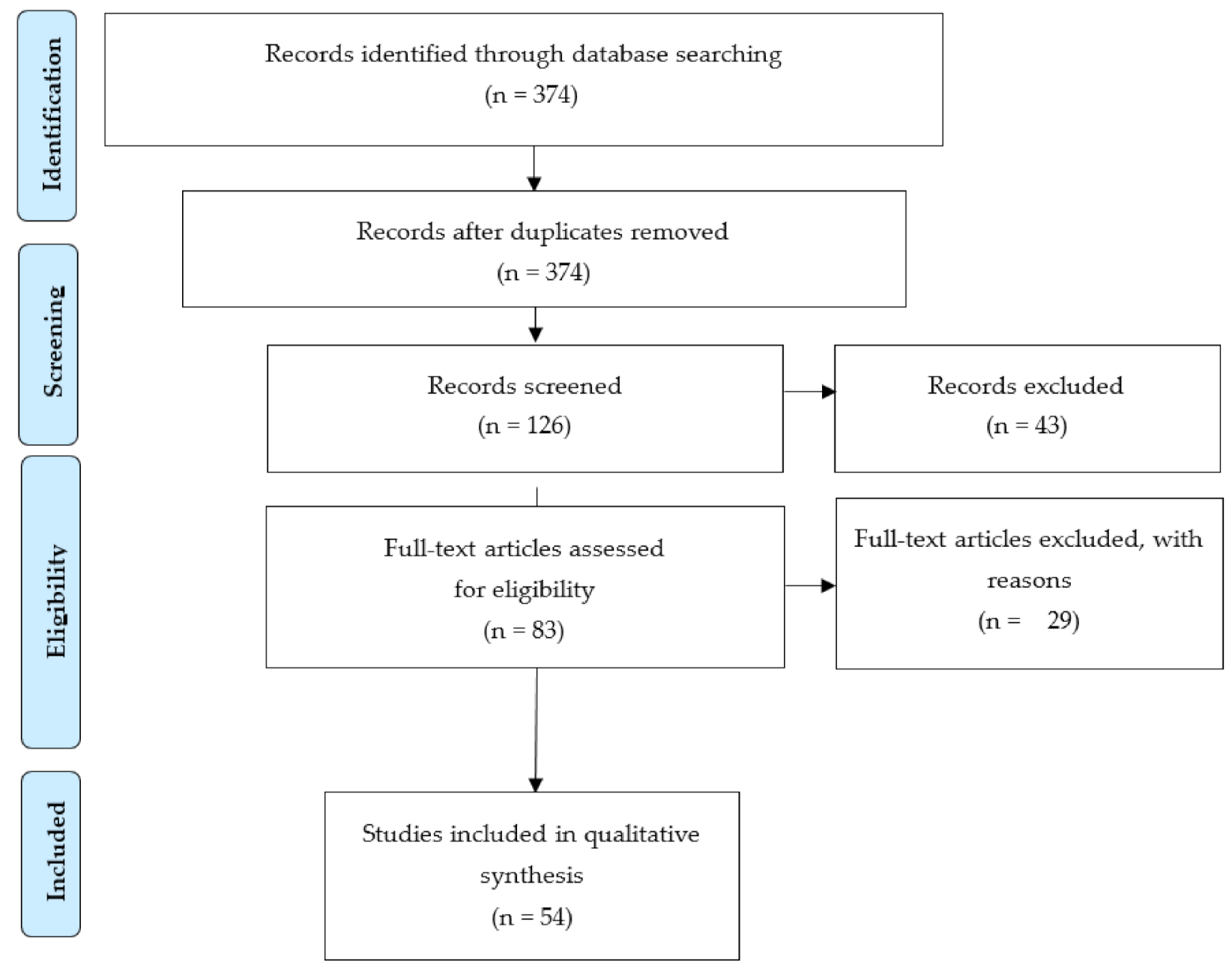

Figure 1. Flowchart of eligibility studies.

Oral complications related to biological therapies have been found, such as lesions at the mucosal level as described in the reviews of the literature by Vigarios et al. [4] (2017) and Watter et al. [23] (2011). Cases of infections at the oral level have been reported in patients treated with adalimumab and infliximab, as described by Cillo et al. [24] (2019) and Filler et al. [25] (2005), as well as a case of exudative erythema multiforme (EEM) in a patient treated with infliximab as described by Edwards et al. [26] (2013). Within the literature, the complication of which more cases have been found is osteonecrosis of the jaws, in patients receiving one or more biological therapies (Figure 2).

Within our search, 49 articles containing a total of 68 cases establish a possible relationship of biological therapies with osteonecrosis of the jaws (Table 1). In $62.69 \%$ of the cases, osteonecrosis was related to denosumab $(16.42 \%)$, an antiresorptive agent; bevacizumab $(28.36 \%)$ or sunitib $(17.91 \%)$, both antiangiogenic agents, coinciding with the three drugs described in 2014 by the AAOMS as producers of MRONJ. 


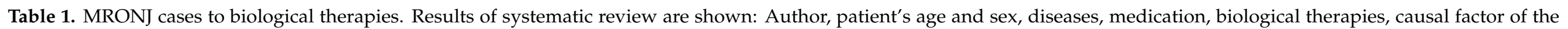
complication, MRONJ stage, and treatment.

\begin{tabular}{|c|c|c|c|c|c|c|c|c|}
\hline Author & Age & Sex & Diseases & Medication & $\begin{array}{l}\text { Biological } \\
\text { Therapies }\end{array}$ & Causal Factor & Mronj Stage & Mronj Treatment \\
\hline \multirow[t]{2}{*}{$\begin{array}{l}\text { Maluf et al. [6] } \\
\quad(2016)\end{array}$} & 69 & M & Lung cancer metastasis & Irinotecan $(\mathrm{QT})$ & Denosumab & Implant surgery & Mandibular ONJ 2 & Surgical \\
\hline & 44 & $\mathrm{~F}$ & $\begin{array}{l}\text { Breast cancer and bone } \\
\text { metastasis }\end{array}$ & Dexamethasone and Fulvestrant & $\begin{array}{l}\text { Denosumab y } \\
\text { Bevacizumab }\end{array}$ & $\begin{array}{l}\text { Endodontic } \\
\text { surgery }\end{array}$ & Mandibular ONJ 2 & Surgical \\
\hline $\begin{array}{l}\text { Sarina et al. [7] } \\
\quad(2013)\end{array}$ & 74 & $\mathrm{M}$ & $\begin{array}{l}\text { Prostate cancer, lung and } \\
\text { bone metastasis }\end{array}$ & $\begin{array}{l}\text { QT, Bicalutamida, Goserelina, Dutasterida, } \\
\text { Calcium y Vitamin D3 y Zoledronic acid }\end{array}$ & Denosumab & - & Mandibular ONJ 2 & Surgical \\
\hline $\begin{array}{l}\text { Kunimichi et al. } \\
\text { [8] (2015) }\end{array}$ & 60 & $\mathrm{~F}$ & Breast cancer & - & Denosumab & $\begin{array}{l}\text { Poorly fitting } \\
\text { prosthesis }\end{array}$ & Mandibular ONJ 1 & - \\
\hline \multirow[t]{4}{*}{$\begin{array}{l}\text { Badr et al. [9] } \\
\quad \text { (2017) }\end{array}$} & 55 & $\mathrm{~F}$ & Breast cancer & - & Denosumab & Tooth extraction & Mandibular ONJ 3 & $\begin{array}{c}\text { Hospitable } \\
\text { treatment }\end{array}$ \\
\hline & 76 & $\mathrm{~F}$ & Breast cancer metastasis & Hormone therapy and radiation therapy & Denosumab & Tooth extraction & Mandibular ONJ 1 & Surgical \\
\hline & 66 & $\mathrm{~F}$ & $\begin{array}{l}\text { Bone, liver and peritoneum } \\
\text { metastasis from breast cancer }\end{array}$ & QT and opioids & Denosumab & Tooth extraction & Mandibular ONJ 3 & Surgical \\
\hline & 66 & $\mathrm{~F}$ & $\begin{array}{l}\text { Bone metástasis and breast } \\
\text { cancer }\end{array}$ & $\begin{array}{l}\text { Hormone therapy and opioids. Prior } \\
\text { zoledronic acid therapy. }\end{array}$ & Denosumab & $\begin{array}{l}\text { Poorly fitting } \\
\text { prosthesis }\end{array}$ & Maxillary ONJ 3 & Conservative \\
\hline $\begin{array}{l}\text { Nifosi et al. [10] } \\
\quad \text { (2017) }\end{array}$ & 47 & M & Renal adenocarcinoma & Calcium and Vitamin D & $\begin{array}{l}\text { Temsirolimus y } \\
\text { Denosumab }\end{array}$ & - & Mandibular ONJ 2 & Conservative \\
\hline $\begin{array}{l}\text { Allegra et al. } \\
\text { [11] (2014) }\end{array}$ & 56 & M & $\begin{array}{c}\text { Waldenstron's } \\
\text { macroglobulinemia, POEMS } \\
\text { syndrome, } \\
\text { lymphoplasmacytic } \\
\text { lymphoma }\end{array}$ & $\begin{array}{l}\text { QT (Doxorrubicin, Cyclophosphamide, } \\
\text { Vincristine, Prednisone) }\end{array}$ & Rituximab & Tooth extraction & Mandibular ONJ 2 & Conservative \\
\hline $\begin{array}{l}\text { Keribin et al. [5] } \\
\quad(2017)\end{array}$ & 69 & M & Nephropathy IgA & $\begin{array}{c}\text { Immunoglobulin, Ranitidine, Trimethoprim } \\
\text { + Sulfamethoxazole, } \\
\text { Valganciclovir, Ofloxacin, Itraconozanol, } \\
\text { Manidipine, Nebivolo, Perindopril, } \\
\text { Indapamide }\end{array}$ & $\begin{array}{l}\text { Rituximab y } \\
\text { Tacrolimus }\end{array}$ & - & $\begin{array}{l}\text { Maxillary and } \\
\text { mandibular ONJ } 2\end{array}$ & Surgical \\
\hline $\begin{array}{l}\text { Baur et al. [12] } \\
\quad(2012)\end{array}$ & 58 & M & Lymphoma cells B & $\begin{array}{c}\text { Chemotherapy } \\
\text { (Doxorrubicin, Cyclophosphamide, } \\
\text { Vincristine, Prednisone), Diltiazem, } \\
\text { Atorvastatin, Meloxicam, Lisinopril, } \\
\text { Hydroclorothiazide, Insulina, Metformin } \\
\text { and Aspirin. }\end{array}$ & Rituximab & - & Mandibular ONJ 2 & Surgical \\
\hline
\end{tabular}


Table 1. Cont.

\begin{tabular}{|c|c|c|c|c|c|c|c|c|}
\hline Author & Age & Sex & Diseases & Medication & $\begin{array}{l}\text { Biological } \\
\text { Therapies }\end{array}$ & Causal Factor & Mronj Stage & Mronj Treatmen \\
\hline $\begin{array}{l}\text { Javelot et al. [13] } \\
\quad(2020)\end{array}$ & 69 & $\mathrm{~F}$ & Rheumatoid arthritis & $\begin{array}{l}\text { Prednisolone, Leflunomid, Valsartan y } \\
\text { Vitamin D3. Previous treatment with } \\
\text { Risedronate and Infliximab. }\end{array}$ & Rituximab & $\begin{array}{c}\text { Herpetic } \\
\text { gingivostomatitis }\end{array}$ & Mandibular ONJ 2 & Surgical \\
\hline $\begin{array}{l}\text { Weigert et al. } \\
\text { [14] (2014) }\end{array}$ & 39 & M & Non- Hogdkin lymphoma & $\begin{array}{c}\text { Ciclofosfamide, Vincristine, Doxorrubicin y } \\
\text { Prednisone }\end{array}$ & Rituximab & - & Mandibular ONJ 0 & Conservative \\
\hline $\begin{array}{l}\text { Manzie et al. } \\
\text { [15] (2019) }\end{array}$ & 76 & $\mathrm{~F}$ & Breast cancer metastasis & $\begin{array}{l}\text { Chemotherapy, Carboplatin, Gemcitabine, } \\
\text { Epirubicine, Paclitaxel, Amlodipine, } \\
\text { Irebsartan }\end{array}$ & $\begin{array}{c}\text { Trastuzumab }+ \\
\text { Pertuzumab }\end{array}$ & - & Mandibular ONJ 2 & Surgical \\
\hline $\begin{array}{l}\text { Mauceri et al. } \\
\text { [16] (2019) }\end{array}$ & 58 & M & $\begin{array}{l}\text { Hurtle's cell carcinoma, lung } \\
\text { metastasis }\end{array}$ & $\begin{array}{l}\text { Radiotherapy, chemotherapy (Doxorrubicin, } \\
\text { Cisplatin), Ciclofosfamide, Metrotexate }\end{array}$ & Lenvatinib & Tooth extraction & Maxillary ONJ 1 & Conservative \\
\hline $\begin{array}{l}\text { Fleissig et al. } \\
\text { [17] (2012) }\end{array}$ & 58 & $\mathrm{~F}$ & $\begin{array}{l}\text { Renal carcinoma, } \\
\text { Osteoporosis }\end{array}$ & Levothyroxine sodium & Sunitinib & Tooth extraction & Mandibular ONJ 2 & Conservative \\
\hline $\begin{array}{l}\text { Brunello et al. } \\
\text { [18] (2009) }\end{array}$ & 59 & M & $\begin{array}{l}\text { Renal carcinoma with ischial } \\
\text { and pulmonary metastasis }\end{array}$ & Previous zoledronic acid & Sunitinib & - & Mandibular ONJ 2 & Conservative \\
\hline $\begin{array}{l}\text { Koch et al. [19] } \\
\quad \text { (2011) }\end{array}$ & 59 & M & $\begin{array}{l}\text { Renal carcinoma, soft tissues } \\
\text { metastasis }\end{array}$ & $\begin{array}{l}\text { Interferon, Vinblastine, Ramipril, } \\
\text { Hydroclorothiazide, Metropolol, } \\
\text { Levothyroxine }\end{array}$ & Sorafenib y Sunitib & Tooth extraction & Mandibular ONJ 1 & Surgical \\
\hline \multirow[t]{2}{*}{$\begin{array}{l}\text { Nicolatou- } \\
\text { Galitis et al. [21] } \\
\quad(2012)\end{array}$} & 19 & $\mathrm{~F}$ & Renal carcinoma & Cisplatin & $\begin{array}{l}\text { Sunitinib y } \\
\text { Everolimus }\end{array}$ & - & Mandibular ONJ 2 & Conservative \\
\hline & 64 & $\mathrm{~F}$ & Renal carcinoma & $\begin{array}{l}\text { Prednisolone, } \\
\text { L-4 }\end{array}$ & Sunitinib & Prosthesis trauma & Mandibular ONJ 2 & Surgical \\
\hline \multirow[t]{2}{*}{$\begin{array}{l}\text { Agrillo et al. [22] } \\
\qquad(2012)\end{array}$} & 65 & M & Renal carcinoma & Previous zoledronic acid & Sunitinib & - & ONJ 3 maxilar & Surgical \\
\hline & 67 & M & Renal carcinoma & Previous zoledronic acid & $\begin{array}{l}\text { Sunitinib y } \\
\text { Temsirolimus }\end{array}$ & - & Mandibular ONJ 3 & Surgical \\
\hline \multirow[t]{2}{*}{$\begin{array}{l}\text { Estilo et al. [27] } \\
\qquad(2008)\end{array}$} & 33 & $\mathrm{~F}$ & Glioblastoma multiforme & Radiotherapy, Temozolomide & Bevacizumab & - & Mandibular ONJ 2 & - \\
\hline & 51 & $\mathrm{~F}$ & Infiltrating ductal carcinoma & $\begin{array}{l}\text { Chemotherapy(Doxorrubicin+ciclofosfamide, } \\
\text { hormonal therapy, Paclitaxel, radiotherapy, } \\
\text { Capecitabine }\end{array}$ & Bevacizumab & - & Mandibular ONJ 2 & Surgical \\
\hline
\end{tabular}


Table 1. Cont.

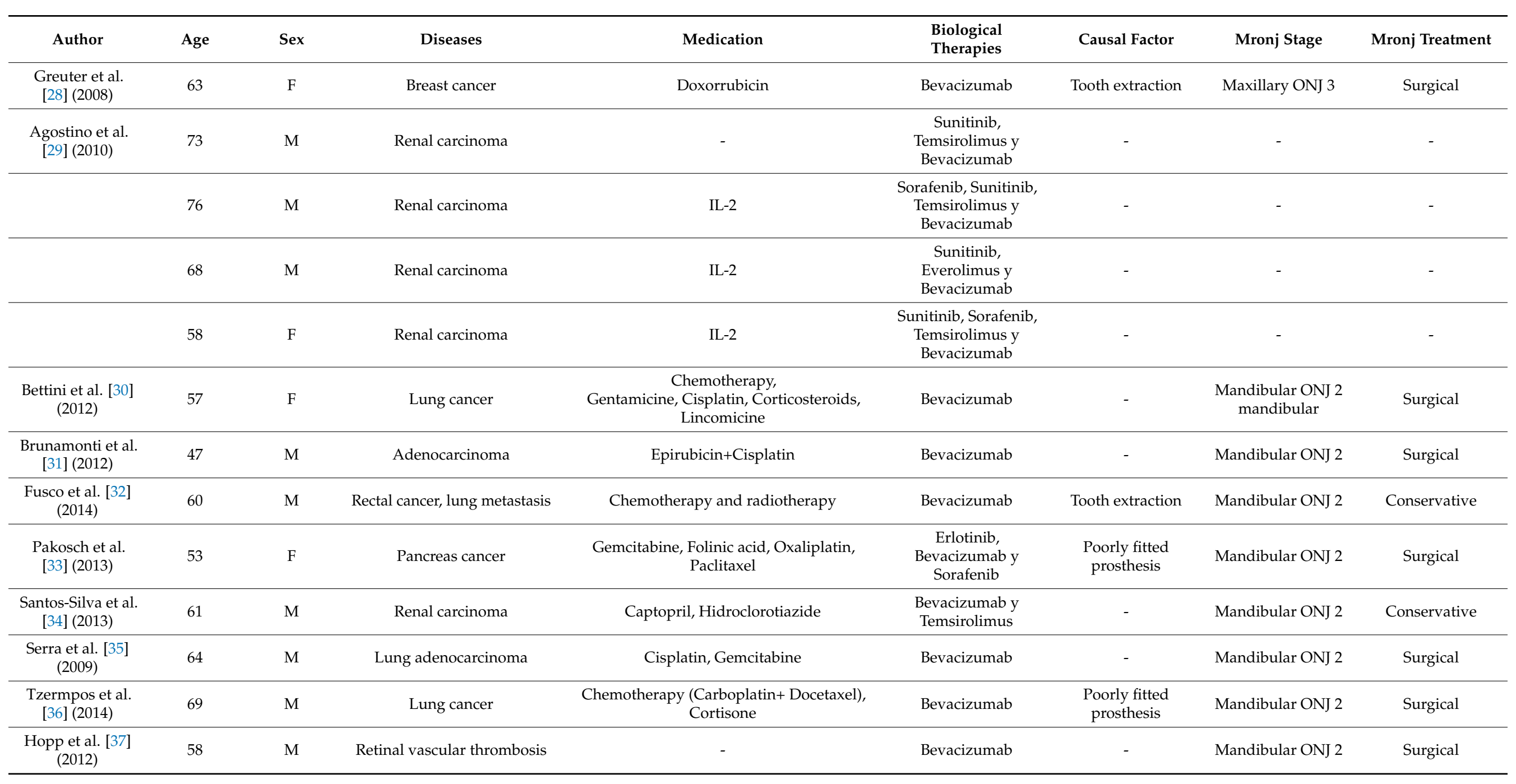


Table 1. Cont.

\begin{tabular}{|c|c|c|c|c|c|c|c|c|}
\hline Author & Age & Sex & Diseases & Medication & $\begin{array}{l}\text { Biological } \\
\text { Therapies }\end{array}$ & Causal Factor & Mronj Stage & Mronj Treatmen \\
\hline $\begin{array}{l}\text { Erovigni et al. } \\
\text { [38] (2016) }\end{array}$ & 79 & $\mathrm{M}$ & Colon adenocarcinoma & $\begin{array}{l}\text { Capecitabin, Oxaliplatin, Fluoracil, Folínic } \\
\text { acid, Irinotecan, radiotherapy }\end{array}$ & Bevacizumab & Tooth extraction & Mandibular ONJ 2 & Conservative \\
\hline \multirow[t]{3}{*}{$\begin{array}{l}\text { Mawardi et al. } \\
\text { [44] (2016) }\end{array}$} & 43 & M & $\begin{array}{c}\text { Metastasis of colorectal } \\
\text { carinoma }\end{array}$ & 5-fluorouracil, Leucovorin, Irinotecan & Aflibercept & - & Mandibular ONJ 1 & Surgical \\
\hline & 63 & M & $\begin{array}{l}\text { Gastrointestinal metastatuc } \\
\text { carcinoid }\end{array}$ & 5-fluorouracil, Leucovorin and Oxaliplatin & Aflibercept & - & Mandibular ONJ 1 & Conservative \\
\hline & 51 & M & $\begin{array}{l}\text { Esophageal carcinoma } \\
\text { metastasis }\end{array}$ & - & Aflibercept & Tooth extraction & Mandibular ONJ 1 & Conservative \\
\hline $\begin{array}{l}\text { Ponzetti et al. } \\
\text { [45] (2016) }\end{array}$ & 64 & $\mathrm{~F}$ & $\begin{array}{l}\text { Metastasis of colorectal } \\
\text { carinoma }\end{array}$ & 5-fluoracil, Irinotecan and Levofolinic Acid & Aflibercept & - & Mandibular ONJ 1 & Conservative \\
\hline $\begin{array}{l}\text { Zarringhalam } \\
\text { et al. [46] (2017) }\end{array}$ & 47 & M & $\begin{array}{l}\text { Intestine, liver, peritoneum } \\
\text { and pelvis metastasis }\end{array}$ & - & Aflibercept & - & Mandibular ONJ 1 & Conservative \\
\hline $\begin{array}{l}\text { Viviano et al. } \\
\text { [47] (2017) }\end{array}$ & 72 & M & $\begin{array}{l}\text { Gastrointestinal stromal } \\
\text { tumor }\end{array}$ & - & Imatinib & Tooth extraction & Mandibular ONJ 2 & Surgical \\
\hline $\begin{array}{l}\text { Kim et al. [40] } \\
\quad(2013)\end{array}$ & 52 & M & $\begin{array}{l}\text { Bone metastasis from thyroid } \\
\text { cancer }\end{array}$ & Previous zoledronc acid treatment & Everolimus & - & Mandibular ONJ 2 & Conservative \\
\hline $\begin{array}{l}\text { Yamamoto et al. } \\
\text { [41] (2017) }\end{array}$ & 80 & $\mathrm{~F}$ & Breast cancer & $\begin{array}{c}\text { Capecitabine, Tamoxifen, Fulvestrant y } \\
\text { Exemestan }\end{array}$ & Everolimus & - & Mandibular ONJ 2 & Conservative \\
\hline $\begin{array}{l}\text { Jung et al. [42] } \\
\text { (2017) }\end{array}$ & 62 & $\mathrm{~F}$ & Renal carcinoma & - & $\begin{array}{c}\text { Everolimus y } \\
\text { Pazopanib }\end{array}$ & Implant surgery & Mandibular ONJ 2 & Surgical \\
\hline $\begin{array}{l}\text { Giancola et al. } \\
\text { [43] (2013) }\end{array}$ & 64 & M & Renal carcinoma metastasis & Previous zoledronc acid treatment & Everolimus & - & Mandibular ONJ 2 & Surgical \\
\hline $\begin{array}{l}\text { Garuti et al. [51] } \\
\quad(2017)\end{array}$ & 74 & M & Hepatic cancer & - & Sorafenib & Tooth extraction & Mandibular ONJ 1 & Conservative \\
\hline $\begin{array}{l}\text { Marino et al. } \\
\text { [49] (2015) }\end{array}$ & 51 & $\mathrm{~F}$ & $\begin{array}{l}\text { Liver and lymph nodes } \\
\text { metástasis from thyroid } \\
\text { cancer }\end{array}$ & $\begin{array}{c}\text { Levotiroxine, Calcitriol, Vitamin D3, } \\
\text { Duloxetine, Propanolol, Lansoprazole y } \\
\text { Loperamide }\end{array}$ & Cabozantinib & Tooth extraction & Mandibular ONJ 2 & Surgical \\
\hline
\end{tabular}


Table 1. Cont

\begin{tabular}{|c|c|c|c|c|c|c|c|c|}
\hline Author & Age & Sex & Diseases & Medication & $\begin{array}{l}\text { Biological } \\
\text { Therapies }\end{array}$ & Causal Factor & Mronj Stage & Mronj Treatmen \\
\hline \multirow[t]{7}{*}{$\begin{array}{l}\text { Mohamed et al. } \\
\text { [39] (2018) }\end{array}$} & 70 & $\mathrm{M}$ & Renal carcinoma & - & $\begin{array}{l}\text { Sunitinib, } \\
\text { Everolimus, } \\
\text { Pazopanib }\end{array}$ & Tooth extraction & Mandibular ONJ 1 & Conservative \\
\hline & 53 & $\mathrm{~F}$ & Lung cancer & $\begin{array}{c}\text { Carboplatin, Cisplatin, Vironelbine } \\
\text { Zoledronic }\end{array}$ & $\begin{array}{l}\text { Erlotinib, Afatinib y } \\
\text { Denosumab }\end{array}$ & Tooth extraction & Maxillary ONJ 2 & Surgical \\
\hline & 55 & $\mathrm{~F}$ & Lung cancer & Carboplatine, Vironelbine, Alimeta. & Bevacizumab & Tooth extraction & Maxillary ONJ 1 & Conservative \\
\hline & 66 & $\mathrm{M}$ & Multiforme glioblastoma & Temozolomide, Irinotecan & Bevacizumab & $\begin{array}{l}\text { Trauma by } \\
\text { intraoral } \\
\text { radiography }\end{array}$ & Mandibular ONJ 2 & Conservative \\
\hline & 74 & $\mathrm{~F}$ & Multiple myeloma & $\begin{array}{c}\text { Cyclophosphamide, Thalidomide, Alkaran, } \\
\text { Capecitabine, Oxaliplatin, Folfiri, Irinotecan, } \\
\text { and Pamidronate }\end{array}$ & $\begin{array}{l}\text { Bevacizumab y } \\
\text { Cetuximab }\end{array}$ & Spontaneous & Mandibular ONJ 1 & Surgical \\
\hline & 53 & $\mathrm{~F}$ & Leukemia & Citarabine and Vincristine & Nilotinib y Dasanitib & Gingival ulcer & Mandibular ONJ 2 & Surgical \\
\hline & 81 & $\mathrm{~F}$ & Leukemia & Capecitabine, Alendronate & $\begin{array}{l}\text { Trastuzumab, } \\
\text { Imatinib y } \\
\text { Denosumab }\end{array}$ & Tooth extraction & Mandibular ONJ 2 & Surgical \\
\hline $\begin{array}{l}\text { Antonuzzo et al. } \\
\text { [53] (2016) }\end{array}$ & 59 & $\mathrm{~F}$ & Metastatic colorectal cancer & - & Regorafenib & - & Maxillary ONJ 3 & - \\
\hline $\begin{array}{l}\text { Preidl et al. [54] } \\
\quad \text { (2014) }\end{array}$ & 36 & $\mathrm{~F}$ & Crohn disease & $\begin{array}{l}\text { Steroids, Risedronate, Zoledronic acid, } \\
\text { Prednisolone }\end{array}$ & Adalimumab & Tooth extraction & Mandibular ONJ 3 & Surgical \\
\hline $\begin{array}{l}\text { Cassoni et al. } \\
\text { [55] (2016) }\end{array}$ & 63 & $\mathrm{~F}$ & Idiopathic arthritis & Salazopyrin & Adalimumab & Implant surgery & $\begin{array}{l}\text { Mandibular ONJ } \\
\text { mandibular }\end{array}$ & Surgical \\
\hline $\begin{array}{l}\text { Favia et al. [56] } \\
\quad(2017)\end{array}$ & 49 & $\mathrm{~F}$ & Chron disease & - & Infliximab & Tooth extraction & $\begin{array}{c}\text { Mandibular ONJ } 3 \\
\text { mandibular }\end{array}$ & Surgical \\
\hline \multirow[t]{2}{*}{$\begin{array}{l}\text { Ekber et al. [50] } \\
\qquad(2013)\end{array}$} & 74 & $\mathrm{~F}$ & $\begin{array}{c}\text { Rheumatoid arthritis and } \\
\text { osteoporosis }\end{array}$ & $\begin{array}{l}\text { Methotrexate, Prednisolone, Folic Acid, } \\
\text { Ibuprofen, Pantoprazole, Calcium, Vit. D. } \\
\text { Previous treatment with zoledronic acid. }\end{array}$ & Infliximab & Tooth extraction & Mandibular ONJ 3 & Surgical \\
\hline & & & & & Tocilizumab & Tarthrectomy & Mandibular ONJ 3 & Surgical \\
\hline
\end{tabular}




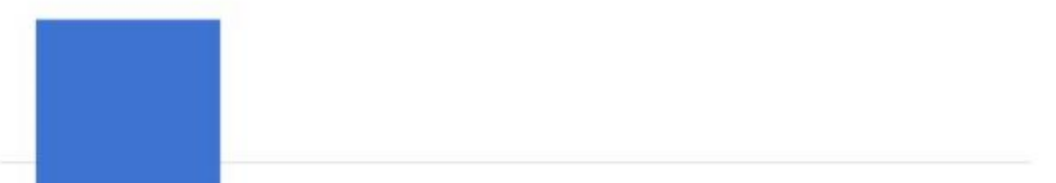

0

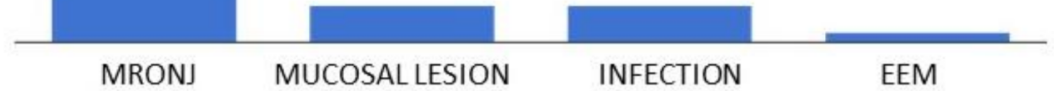

Figure 2. Percentage of oral complications related to biological therapy. MRONJ is the most common oral complication registered, followed by mucosal lesion, infection, and exudative erythema multiforme.

Among them, it can be observed that there are more cases of osteonecrosis in the mandible (83.58\%) than in the maxilla, as well as a higher frequency in men, with $53.73 \%$. Regarding the treatment carried out for osteonecrosis, it can be observed that a surgical treatment was carried out in $55.22 \%$ of the cases, which consists in the majority of them in a mandibular sequestrectomy or resection. Conservative treatment consists mainly of antibiotic therapy, antimicrobial rinses, strict oral hygiene measures, as well as modifications in biological therapy.

\section{Discussion}

Although osteonecrosis is the most studied complication due to its severity, there are also references to other complications at the oral level. Although these complications do not involve such a high risk for the patient, they require treatment to control and improve their quality of life.

These drugs can be difficult to identify clinically by their brand names. A table (Table 2) is presented with the drugs and their most common commercial name to facilitate their identification for the professional, as well as a series of recommendations on the management of patients with biological therapy.

Table 2. Biological therapies associated with oral complications and trade name. Most common biological therapies and trade name related to oral complications are shown.

\begin{tabular}{ccc}
\hline Drug & Trade Name & Category \\
\hline Adalimumab & Humira & MAB \\
Afatinib & Giotrif & TKI \\
Aflibercept & Zaltrap, Eylea & Fusion prothein \\
Axitinib & Inlyta & TKI \\
Bevacizumab & Avastin & MAB \\
Cabozantinib & Cometriq, Cabometyx & TKI \\
Dasatinib & Sprycel & TKI \\
\hline
\end{tabular}


Table 2. Cont.

\begin{tabular}{ccc}
\hline Drug & Trade Name & Category \\
\hline Denosumab & Xgeva, Prolia & MAB \\
Erlotinib & Tarceva & TKI \\
Everolimus & Afinitor & mTOR inhibitor \\
Imatinib & Gleevec & TKI \\
Infliximab & Remicade & MAB \\
Ipilimumab & Yervoy & MAB \\
Lenvatinib & Lenvima & TKI \\
Nilotinib & Tasigna & TKI \\
Pazopanib & Votrient & TKI \\
Regorafenib & Stivarga & TKI \\
Rituximab & Erbitux, Rituxan & MAB \\
Sorafenib & Nexavar & TKI \\
Sunitinib & Sutent & TKI \\
Tacrolimus & Advagraf & mTOR inhibitor \\
Temsirolimus & Torisel & mTOR inhibitor \\
Tocilizumab & Actembra & MAB \\
Trastuzumab+Pertuzumab & Herceptin + Perjeta & MAB \\
\hline TKI: tyrosine-kinase inhibitor, MAB: monoclonal antibody, mTOR: mammalian target of rapamycin.
\end{tabular}

TKI: tyrosine-kinase inhibitor, MAB: monoclonal antibody, mTOR: mammalian target of rapamycin.

\subsection{Management of Patients Undergoing Treatment with Biological Therapies}

The management of patients undergoing treatment with these biological therapies requires monitoring and special measures by the dentist to avoid the adverse effects previously described.

Before starting therapy, it is essential to carry out a complete dental treatment. Dental treatment includes a comprehensive dental examination with orthopantomography and intraoral radiographs, extraction of teeth with impossible prognosis, conservative dental treatments and periodontal interventions, prosthesis adjustment, factor control risks such as tobacco and diabetes, as well as patient education on the importance of oral care.

Infection is one of the most important adverse effects, but not enough scientific evidence has been found to support the use of antibiotic prophylaxis in these patients when performing surgery. The considerations prior to a surgical treatment that we will have to take into account are [1]:

Complete blood count if the procedure involves bleeding.

Prothrombin time (PT) and INR, if the patient has liver damage. This is one of the possible adverse effects in long-term treatment with biological therapies [57].

Interruption of treatment with biological agents before the procedure. The so-called drug holiday is a subject of controversy between different authors. With bisphosphonates, drug holiday protocols exist before performing invasive surgical treatment, although continuing with the controversy, a recent study in Japan [58] suggests that the withdrawal of BPs would not reduce the risk of developing adverse effects after surgery such as osteonecrosis [59]. This may be due to the fact that BPs have a long-lasting skeletal retention, while biological drugs such as denosumab have a reversible effect. For this reason, the use of drug holiday in patients receiving denosumab could be endorsed. However, the temporary suspension of medications must be compatible with the underlying pathology and be authorized by the prescriber. To date, there is insufficient scientific evidence to confirm the effectiveness of the drug holiday [60,61].

Regarding the surgical technique used in this type of patients, it should be as atraumatic as possible and can be accompanied by the use of plasma rich in growth factors (PRGF) to promote bone and soft tissue regeneration after surgery, reducing thus the risk of infection, and the time of exposure of the wound to bacteria. After surgery, experts recommend the use of antimicrobial rinses and systemic antibiotics. 


\subsection{Management of Complications Associated with Biological Therapies}

Oral toxicities are one of the adverse effects in treatment with biological therapies. The management of these oral toxicities consists of:

Mucositis, stomatitis, and aphthous lesions: Basic oral care, corticosteroids (topical, intralesional, or oral), morphine rinses, systemic analgesics, LLLT (Low Level Laser Therapy), and change in medication and interruption of previously agreed radiotherapy sessions with the oncologist.

Hyperkeratotic lesions and dyschromia of the mucosa: Monthly reviews and biopsy in case of suspicion. lesions.

Geographic tongue: Avoidance of irritants, rinsing with corticosteroids in painful

Dysgeusia: Nutritional recommendations such as abundant consumption of liquids and cold meals; Changes in the medication previously agreed with the dentist can be proposed.

Lichenoid lesions: Topical corticosteroids for painful lesions and periodic reviews, as well as change in medication.

Telangiectasias and mucosal bleeding: Basic oral care and change in medication.

Xerostomía: Basic oral care, hydration, use of sialogogues such as pilocarpine or artificial salivary substitutes (palliative).

Dysestesia: Basic oral care, avoidance of irritants and symptomatic treatment with analgesics. In case of neuropathy, use drugs such as clonazepam or gabapentin.

MRONJ: This complication is divided by the AAOMS into four grades with a specific treatment for each of them [2]. (Table 3)

Table 3. Complication grades divided by the AAOMS and specific treatment. Complication grades according to AAOMS and recommended treatment are shown.

\begin{tabular}{|c|c|}
\hline Grade & Treatment \\
\hline Risk: No apparent necrotic bone & Treatment not indicated, patient education \\
\hline $\begin{array}{l}\text { Grade 0: No clinical evidence of bone necrosis, but nonspecific } \\
\text { clinical findings, radiographic changes, and symptoms. }\end{array}$ & $\begin{array}{l}\text { Systemic management, including the use of analgesics and } \\
\text { antibiotic. }\end{array}$ \\
\hline $\begin{array}{l}\text { Grade 1: Exposure and bone necrosis, or fistulas that can be probed to } \\
\text { bone in asymptomatic patients without evidence of infection. }\end{array}$ & Antibacterial rinses, clinical follow-up, patient education. \\
\hline $\begin{array}{l}\text { Grade 2: Exposure and bone necrosis, or fistulas that can be probed to } \\
\text { the bone, associated with infection with pain or erythema in the } \\
\text { region of exposed bone with or without purulent drainage. }\end{array}$ & $\begin{array}{l}\text { Symptomatic treatment with oral antibiotics, antibacterial } \\
\text { rinses, pain control, debridement to relieve soft tissue } \\
\text { irritation and control infection. }\end{array}$ \\
\hline $\begin{array}{l}\text { Grade 3: Presence of one of the following signs: bone exposure and } \\
\text { necrosis that extends beyond the alveolar region, pathological } \\
\text { fractures, extraoral fistulas, oro-nasal or oro-antral communication, } \\
\text { osteolysis that extends to the lower border of the mandible or floor of } \\
\text { the breast. }\end{array}$ & $\begin{array}{c}\text { Antibacterial rinses, antibiotic therapy and pain control, } \\
\text { surgical debridement or resection to alleviate infection and } \\
\text { long-lasting pain. }\end{array}$ \\
\hline
\end{tabular}

It has been observed that the use of hyperbaric oxygen therapy as an adjunct to surgical and non-surgical treatment produces improvements in wound healing, pain, and quality of life. However, there are no statistically significant differences with respect to the control group $[2,62]$.

Other studies mention the use of low-frequency lasers, parathyroid hormone and BMPs (Bone Morphogenetic Protein) as strategies in non-surgical treatment, but more studies are needed to verify their efficacy [2].

\subsection{Study Limitations}

The present study meets a series of limitations: There is a lack of scientific evidence about the possible adverse effects that these drugs may present at the oral level, in addition, it is difficult to relate them to a single drug in polymedicated patients. Likewise, in most 
cases, the adverse event is not easily related to dental treatment due to the time elapsed until it appears.

Despite this, this review presents all the oral adverse events reported to date in relation to these drugs.

\section{Conclusions}

The biological drugs that, according to the literature described, have the greatest relationship with adverse events in the dental field are bevacizumab, denosumab and sunitinib. The main adverse event is osteonecrosis of the jaws (MRONJ), resolved in most of the cases described with surgical treatment.

It is necessary to take into account the oral complications associated with these therapies and the importance of monitoring by the dentist from the beginning, in order to prevent or reduce the appearance of these.

Despite the reported cases, more studies are needed to describe in a more exhaustive way the appearance of complications that could be related to these treatments.

Author Contributions: Conceptualization, M.A.-B. and C.Á.-G.; methodology, C.C.-V; data selection, I.L.-B., L.M.S.-A. and C.M.-G.; data extraction, M.A.-B.; writing-original draft preparation, C.Á.-G.; writing-review and editing, C.C.-V.; visualization, C.M.-G.; supervision, L.M.S.-A.; submission, C.C.V. All authors have read and agreed to the published version of the manuscript.

Funding: This research received no external funding.

Institutional Review Board Statement: Not applicable.

Informed Consent Statement: Not applicable.

Conflicts of Interest: The authors declare no conflict of interest.

\section{References}

1. Radfar, L.; Ahmadabadi, R.E.; Masood, F.; Scofield, R.H. Biological therapy and dentistry: A review paper. Oral Surg. Oral Med. Oral Pathol. Oral Radiol. 2015, 120, 594-601. [CrossRef] [PubMed]

2. Ruggiero, S.L.; Dodson, T.B.; Fantasia, J.; Goodday, R.; Aghaloo, T.; Mehrotra, B.; O'Ryan, F. American Association of Oral and Maxillofacial Surgeons Position Paper on Medication-Related Osteonecrosis of the Jaw-2014 Update. J. Oral Maxillofac. Surg. 2014, 72, 1938-1956. [CrossRef]

3. Yarom, N.; Shapiro, C.L.; Peterson, D.E.; Van Poznak, C.H.; Bohlke, K.; Ruggiero, S.L.; O’Ryan, F. Medication-Related Osteonecrosis of the Jaw: MASCC/ISOO/ASCO Clinical Practice Guideline. J. Clin. Oncol. 2019, 37, 2270-2290. [CrossRef]

4. Vigarios, N.P.; Epstein, J.B.; Sibaud, V. Oral mucosal changes induced by anticancer targeted therapies and immune checkpoint inhibitors. Support Care Cancer 2017, 25, 1713-1739.

5. Keribin, P.; Guerrot, D.; Jardin, F.; Moizan, H. Osteonecrosis of the Jaw in a Patient Presenting with Post-Transplantation Lymphoproliferative Disorder Treated With Rituximab: A Case Report. J. Oral Maxillofac. Surg. 2017, 75, 2599-2605. [CrossRef]

6. Maluf, G.; Pinho, M.C.D.; Cunha, S.R.D.B.D.; Santos, P.S.D.S.; Fregnani, E.R. Surgery Combined with LPRF in Denosumab Osteonecrosis of the Jaw: Case Report. Braz. Dent. J. 2016, 27, 353-358. [CrossRef]

7. Pichardo, S.E.C.; Kuypers, S.C.C.; van Merkesteyn, J.P.R. Denosumab osteonecrosis of the mandible: A new entity? A case report. J. Craniomaxillofac. Surg. 2013, 41, e65-e69. [CrossRef]

8. Niibe, K.; Ouchi, T.; Iwasaki, R.; Nakagawa, T.; Horie, N. Osteonecrosis of the jaw in patients with dental prostheses being treated with bisphosphonates or denosumab. J. Prosthodont. Res. 2015, 59, 3-5. [CrossRef]

9. Badr, M.; Kyriakidou, E.; Atkins, S.; Harrison, S. Aggressive denosumab-related jaw necrosis-A case series. Br. Dent. J. 2017, 223, 13-16. [CrossRef] [PubMed]

10. Nifosi, A.F.; Nifosi, L.; Nifosi, G. Osteonecrosis of the Jaw in a patient treated with denosumab and temsirolimus. SAJ Case Rep. 2017, 4, 1-4.

11. Allegra, A.; Oteri, G.; Alonci, A.; Bacci, F.; Penna, G.; Minardi, V.; Maisanoa, V.; Musolino, C. Association of osteonecrosis of the jaws and POEMS syndrome in a patient assuming rituximab. J. Craniomaxillofac. Surg. 2014, 42, 279-282. [CrossRef] [PubMed]

12. Baur, D.A.; Weber, J.M.; Collette, D.C.; Dhaliwal, H.; Quereshy, F. Osteonecrosis of the Jaws Unrelated to Bisphosphonate Exposure: A Series of 4 Cases. J. Oral Maxillofac. Surg. 2012, 70, 2802-2808. [CrossRef]

13. Javelot, M.-J.; Sergheraert, J.; Agbo-Godeau, S.; Levy-Weil, F.; Laurence, S.; Goudot, P.; Khonsarie, R.-H.; Mauprivezb, C. Rituximab as a trigger factor of medication-related osteonecrosis of the jaw. A case report. J. Stomatol. Oral Maxillofac. 2020, 121, 300-304. [CrossRef]

14. Weigert, K.; Lewgoy, J.; Mazzoleni, D.S.; Franco, F.R.; Lisandra, E.; Henrique Sasso, J. Rituximab and osteonecrosis of the jaws: Case study. Oral Surg. Oral Med. Oral Pathol. Oral Radiol. 2014, 117, e188-e189. [CrossRef] 
15. Manzie, T.; Vujcich, N.; Chan, A. Osteonecrosis of the jaw in an anti-resorptive naïve patient taking trastuzumab and pertuzumab: Case report. Aust. Dent. J. 2020, 65, 305-307. [CrossRef]

16. Mauceri, R.; Panzarella, V.; Morreale, I.; Campisi, G. Medication-related osteonecrosis of the jaw in a cancer patient receiving lenvatinib. Int. J. Oral Maxillofac. Surg. 2019, 48, 1530-1532. [CrossRef] [PubMed]

17. Fleissig, Y.; Regev, E.; Lehman, H. Sunitinib related osteonecrosis of jaw: A case report. Oral Surg. Oral Med. Oral Pathol. Oral Radiol. 2012, 113, e1-e3. [CrossRef] [PubMed]

18. Brunello, A.; Saia, G.; Bedogni, A.; Scaglione, D.; Basso, U. Worsening of osteonecrosis of the jaw during treatment with sunitinib in a patient with metastatic renal cell carcinoma. Bone 2009, 44, 173-175. [CrossRef]

19. Koch, F.P.; Walter, C.; Hansen, T.; Jäger, E.; Wagner, W. Osteonecrosis of the jaw related to sunitinib. Oral Maxillofac. Surg. 2011, 15, 63-66. [CrossRef] [PubMed]

20. Melloni, C.; Tuttolomondo, A.; Anfosso, A.; Calamia, C.; Clemente, F.D.; Cordova, A. Sunitinib related osteonecrosis of the jaw (SURONJ): A rare occurrence? Eur. J. Plast. Surg. 2016, 39, 161-162. [CrossRef]

21. Nicolatou-Galitis, O.; Schiødt, M.; Mendes, R.A.; Ripamonti, C.; Hope, S.; Drudge-Coates, L.; Niepel, D.; Van den Wyngaert, T. Medication-related osteonecrosis of the jaw: Definition and best practice for prevention, diagnosis, and treatment. Oral Surg. Oral Med. Oral Pathol. Oral Radiol. 2019, 127, 117-135. [CrossRef]

22. Agrillo, A.; Nastro Siniscalchi, E.; Facchini, A.; Filiaci, F.; Ungari, C. Osteonecrosis of the jaws in patients assuming bisphosphonates and sunitinib: Two case reports. Eur. Rev. Med. Pharmacol. Sci. 2012, 16, 952-957.

23. Watters, A.L.; Epstein, J.B.; Agulnik, M. Oral complications of targeted cancer therapies: A narrative literature review. Oral Oncol. 2011, 47, 441-448. [CrossRef]

24. Cillo, J.E.; Barbosa, N. Adalimumab-Related Dental Implant Infection. J. Oral Maxillofac. Surg. 2019, 77, 1165-1169. [CrossRef]

25. Filler, S.G.; Yeaman, M.R.; Sheppard, D.C. Tumor Necrosis Factor Inhibition and Invasive Fungal Infections. Clin. Infect. Dis. 2005, 41, S208-S212. [CrossRef] [PubMed]

26. Edwards, D.; Boritz, E.; Cowen, E.W.; Brown, R.S. Erythema multiforme major following treatment with infliximab. Oral Surg. Oral Med. Oral Pathol. Oral Radiol. 2013, 115, e36-e40. [CrossRef] [PubMed]

27. Estilo, C.L.; Fornier, M.; Farooki, A.; Carlson, D.; Bohle, G.; Huryn, J.M. Osteonecrosis of the Jaw Related to Bevacizumab. J. Clin. Oncol. 2008, 26, 4037-4038. [CrossRef]

28. Greuter, S.; Schmid, F.; Ruhstaller, T.; Thuerlimann, B. Bevacizumab-associated osteonecrosis of the jaw. Ann. Oncol. 2008, 19, 2091-2092. [CrossRef] [PubMed]

29. Agostino, N.M.; Gingrich, R.; Drabick, J.J. Bevacizumab Demonstrates Prolonged Disease Stabilization in Patients with Heavily Pretreated Metastatic Renal Cell Carcinoma: A Case Series and Review of the Literature. Adv. Urol. 2010, 2010, 687043. [CrossRef]

30. Bettini, G.; Blandamura, S.; Saia, G.; Bedogni, A. Bevacizumab-related osteonecrosis of the mandible is a self-limiting disease process. BMJ Case Rep. 2012, 2012, bcr2012007284. [PubMed]

31. Binello, P.B.; Bandelloni, R.; Labanca, M.; Buffoli, B.; Rezzani, R.; Rodella, L.F. Osteonecrosis and the Jaws and Bevacizumab Therapy: A Case Report. Int. J. Immunopathol. Pharmacol. 2012, 25, 789-791. [CrossRef]

32. Campisi, G.; Fedele, S.; Fusco, V.; Pizzo, G.; Di Fede, O.; Bedogni, A. Epidemiology, clinical manifestations, risk reduction and treatment strategies of jaw osteonecrosis in cancer patients exposed to antiresorptive agents. Future. Oncol. 2014, 10, 257-275. [CrossRef] [PubMed]

33. Pakosch, D.; Papadimas, D.; Munding, J.; Kawa, D.; Kriwalsky, M.S. Osteonecrosis of the mandible due to anti-angiogenic agent, bevacizumab. Oral Maxillofac. Surg. 2013, 17, 303-306. [CrossRef] [PubMed]

34. Santos-Silva, A.R.; Belizário Rosa, G.A.; de Castro Júnior., G.; Dias, R.B.; Prado Ribeiro, A.C.; Brandão, T.B. Osteonecrosis of the mandible associated with bevacizumab therapy. Oral Surg. Oral Med. Oral Pathol. Oral Radiol. 2013, 115, e32-36. [CrossRef] [PubMed]

35. Serra, E.; Paolantonio, M.; Spoto, G.; Mastrangelo, F.; Tetè, S.; Dolci, M. Bevacizumab-Related Osteneocrosis of the Jaw. Int. J. Immunopathol. Pharmacol. 2009, 22, 1121-1123. [CrossRef]

36. Tzermpos, F.; Ismail, A.; Pavli, M.; Tosios, K.I. Osteonecrosis of the mandible in a patient with lung adenocarcinoma undergoing anti-angiogenic therapy with bevacizumab: Bevacizumab associated osteonecrosis of the jaw. Oral Surg. 2016, 9, 40-46. [CrossRef]

37. Hopp, R.N.; Pucci, J.; Santos-Silva, A.R.; Jorge, J. Osteonecrosis After Administration of Intravitreous Bevacizumab. J. Oral Maxillofac. Surg. 2012, 70, 632-635. [CrossRef] [PubMed]

38. Erovigni, F.; Gambino, A.; Cabras, M.; Fasciolo, A.; Bianchi, S.; Bellini, E.; Fusco, V. Delayed Diagnosis of Osteonecrosis of the Jaw (ONJ) Associated with Bevacizumab Therapy in Colorectal Cancer Patients: Report of Two Cases. Dent. J. 2016, 4, 39. [CrossRef] [PubMed]

39. Mohamed, H.A.M.; Nielsen, C.E.N.; Schiodt, M. Medication related osteonecrosis of the jaws associated with targeted therapy as monotherapy and in combination with antiresorptives. A report of 7 cases from the Copenhagen Cohort. Oral Surg. Oral Med. Oral Pathol. Oral Radiol. 2018, 125, 157-163. [CrossRef]

40. Kim, D.W.; Jung, Y.-S.; Park, H.-S.; Jung, H.-D. Osteonecrosis of the jaw related to everolimus: A case report. Br. J. Oral Maxillofac. Surg. 2013, 51, e302-e304. [CrossRef] [PubMed]

41. Yamamoto, D.; Tsubota, Y.; Utsunomiya, T.; Sueoka, N.; Ueda, A.; Endo, K.; Yoshikawa, K.; Kon, M. Osteonecrosis of the jaw associated with everolimus: A case report. Mol. Clin. Oncol. 2017, 6, 255-257. [CrossRef] 
42. Jung, T.-Y. Osteonecrosis of jaw after antiangiogenic agent administration in a renal cell carcinoma patient. Oral Maxillofac. Surg. Cases 2017, 3, 27-33. [CrossRef]

43. Giancola, F.; Campisi, G.; Lo Russo, L.; Muzio, L.L.; Di Fede, O. Osteonecrosis of the jaw related to everolimus and bisphosphonate: A unique case report? Ann. Stomatol. 2013, 4, 20-21.

44. Mawardi, H.; Enzinger, P.; McCleary, N.; Manon, R.; Villa, A.; Treister, N.; Woo, S.B. Osteonecrosis of the jaw associated with ziv-aflibercept. J. Gastrointest. Oncol. 2016, 6, e81-e87. [CrossRef]

45. Ponzetti, A.; Pinta, F.; Spadi, R.; Mecca, C.; Fanchini, L.; Zanini, M.; Ciuffreda, L.; Racca, P. Jaw Osteonecrosisassociated with Aflibercept, Irinotecan and Fluorouracil: Attention to Oral District. Tumori 2016, 102, S74-77. [CrossRef] [PubMed]

46. Zarringhalam, P.; Brizman, E.; Shakib, K. Medication-related osteonecrosis of the jaw associated with aflibercept. Br. J. Oral Maxillofac. Surg. 2017, 55, 314-315. [CrossRef] [PubMed]

47. Viviano, M.; Rossi, M.; Cocca, S. A rare case of osteonecrosis of the jaw related to imatinib. J. Korean. Assoc. Oral Maxillofac. Surg. 2017, 43, 120. [CrossRef] [PubMed]

48. Owosho, A.A.; Scordo, M.; Yom, S.K.; Randazzo, J.; Chapman, P.B.; Huryn, J.M.; Estilo, C.L. Osteonecrosis of the jaw a new complication related to Ipilimumab. Oral Oncol. 2015, 51, e100-e101. [CrossRef]

49. Marino, R.; Orlandi, F.; Arecco, F.; Gandolfo, S.; Pentenero, M. Osteonecrosis of the jaw in a patient receiving cabozantinib. Aust. Dent. J. 2015, 60, 528-531. [CrossRef]

50. Ebker, T.; Rech, J.; von Wilmowsky, C.; Neukam, F.W.; Stockmann, P. Fulminant course of osteonecrosis of the jaw in a rheumatoid arthritis patient following oral bisphosphonate intake and biologic therapy. Rheumatology 2013, 52, 218-220. [CrossRef]

51. Garuti, F.; Camelli, V.; Spinardi, L.; Bucci, L.; Trevisani, F. Osteonecrosis of the Jaw during Sorafenib Therapy for Hepatocellular Carcinoma. Tumori 2016, 102, S69-S70. [CrossRef]

52. Patel, V.; Sproat, C.; Kwok, J.; Tanna, N. Axitinib-related osteonecrosis of the jaw. Oral Surg. Oral Med. Oral Pathol. Oral Radiol. 2017, 124, e257-e260. [CrossRef] [PubMed]

53. Antonuzzo, L.; Lunghi, A.; Giommoni, E.; Brugia, M.; Di Costanzo, F. Regorafenib Also Can Cause Osteonecrosis of the Jaw. J. Natl. Cancer. Inst. 2016, 108, djw002. [CrossRef] [PubMed]

54. Preidl, R.H.; Ebker, T.; Raithel, M.; Wehrhan, F.; Neukam, F.W.; Stockmann, P. Osteonecrosis of the jaw in a Crohn's disease patient following a course of Bisphosphonate and Adalimumab therapy: A case report. BMC Gastroenterol. 2014, 14, 6. [CrossRef]

55. Cassoni, A.; Romeo, U.; Terenzi, V.; Della Monaca, M.; Rajabtork Zadeh, O.; Raponi, I.; Fadda, M.T.; Polimeni, A.; Valentini, V. Adalimumab: Another Medication Related to Osteonecrosis of the Jaws? Case. Rep. Dent. 2016, 2016, 1-6. [CrossRef]

56. Favia, G.; Tempesta, A.; Limongelli, L.; Crincoli, V.; Iannone, F.; Lapadula, G.; Maiorano, E. A Case of Osteonecrosis of the Jaw in a Patient with Crohn's Disease Treated with Infliximab. Am. J. Case. Rep. 2017, 18, 1351-1356. [CrossRef]

57. Girolomoni, G.; Altomare, G.; Ayala, F.; Berardesca, E.; Calzavara-Pinton, P.; Chimenti, S.; Peserico, A.; Guerra, A.P.; Vena, G.A. Safety of anti-TNF $\alpha$ agents in the treatment of psoriasis and psoriatic arthritis. Immunopharmacol. Immunotoxicol. 2012, 34, 548-560. [CrossRef] [PubMed]

58. Hasegawa, T.; Kawakita, A.; Ueda, N.; Funahara, R.; Tachibana, A.; Kobayashi, M.; Kondou, E.; Takeda, D.; Kojima, Y.; Sato, S.; et al. A multicenter retrospective study of the risk factors associated with medication-related osteonecrosis of the jaw after tooth extraction in patients receiving oral bisphosphonate therapy: Can primary wound closure and a drug holiday really prevent MRONJ? Osteoporos. Int. 2017, 28, 2465-2473. [CrossRef]

59. Song, M. Dental care for patients taking antiresorptive drugs: A literature review. Restor. Dent. Endod. 2019, 44, e42. [CrossRef]

60. Di Fede, O.; Panzarella, V.; Mauceri, R.; Fusco, V.; Bedogni, A.; Lo Muzio, L.; Board, S.O.; Campisi, G. The Dental Management of Patients at Risk of Medication-Related Osteonecrosis of the Jaw: New Paradigm of Primary Prevention. BioMed. Res. Int. 2018, 2018, 1-10. [CrossRef]

61. Beth-Tasdogan, N.H.; Mayer, B.; Hussein, H.; Zolk, O. Interventions for managing medication-related osteonecrosis of the jaw. Cochrane. Database. Syst. Rev. 2017, 10, CD012432. [CrossRef]

62. Rollason, V.; Laverrière, A.; MacDonald, L.C.I.; Walsh, T.; Tramèr, M.R.; Vogt-Ferrier, N.B. Interventions for treating bisphosphonate-related osteonecrosis of the jaw (BRONJ). Cochrane. Database. Syst. Rev. 2016, 2, CD008455. [CrossRef] [PubMed] 